\title{
Rates and determinants of seasonal influenza vaccination in pregnancy and association with neonatal outcomes
}

\author{
Alexandra Legge BSc, Linda Dodds PhD, Noni E. MacDonald MD, Jeffrey Scott MD, Shelly McNeil MD
}

\begin{abstract}
Background: There is growing evidence that seasonal influenza vaccination in pregnancy has benefits for mother and baby. We determined influenza vaccination rates among pregnant women during the 2 nonpandemic influenza seasons following the $2009 \mathrm{H} 1 \mathrm{~N} 1$ pandemic, explored maternal factors as predictors of influenza vaccination status and evaluated the association between maternal influenza vaccination and neonatal outcomes.
\end{abstract}

Methods: We used a population-based perinatal database in the province of Nova Scotia, Canada, to examine maternal vaccination rates, determinants of vaccination status and neonatal outcomes. Our cohort included women who gave birth between Nov. 1, 2010, and Mar. 31, 2012. We compared neonatal outcomes between vaccinated and unvaccinated women using logistic regression analysis.

Results: Overall, 1958 (16.0\%) of 12223 women in our cohort received the influenza vaccine during their pregnancy. Marital status, parity, location of residence (rural v. urban), smoking during pregnancy and maternal influenza risk status were determinants of maternal vaccine receipt. The odds of preterm birth was lower among infants of vaccinated women than among those of nonvaccinated women (adjusted odds ratio [OR] 0.75, 95\% confidence interval $[\mathrm{Cl}]$ 0.60-0.94). The rate of lowbirth-weight infants was also lower among vaccinated women (adjusted OR $0.73,95 \% \mathrm{Cl}$ 0.56-0.95).

Interpretation: Despite current guidelines advising all pregnant women to receive the seasonal influenza vaccine, influenza vaccination rates among pregnant women in our cohort were low in the aftermath of the 2009 H1N1 pandemic. This study and others have shown an association between maternal influenza vaccination and improved neonatal outcomes, which supports stronger initiatives to promote vaccination during pregnancy.
I nfluenza viruses are the leading cause of serious wintertime respiratory morbidity worldwide. Several studies investigating the effects of influenza-related illness during pregnancy have shown a strong impact on the health of pregnant women in terms of increased rates of hospital admission because of respiratory illness. ${ }^{1-3}$ Schanzer and colleagues ${ }^{2}$ found that pregnant women in Canada were at increased risk of influenza-related hospital admission when compared with nonpregnant women of similar age and health status. In addition, influenza-related illness during pregnancy may have a negative impact on neonatal outcomes. A study in Nova Scotia, Canada, showed that infants whose mothers were admitted to hospital because of respiratory illness during influenza season while pregnant were more likely to be small for gestational age and to have lower mean birth weight. ${ }^{4}$

By 2007, the cumulative evidence from these and other studies was compelling enough for advisory boards in Canada to recommend rou- tine influenza vaccination for all pregnant women, including those without medical comorbidities. ${ }^{5}$ Despite these recommendations, seasonal vaccination rates among pregnant women have remained low. In a cohort of pregnant women who delivered at the IWK Health Centre, Halifax, from 2006 to 2009 , only $20 \%$ had received the vaccine during their pregnancy. ${ }^{6}$ Increased vaccination rates among pregnant women were reported for the 2009 H1N1 pandemic year, ${ }^{7}$ but it is unknown whether this has translated into higher rates of seasonal influenza vaccination since then. Studies have shown that concern about vaccine safety is the most commonly cited reason for refusing the vaccine,,$^{8,9}$ despite much evidence showing it to be safe in pregnancy. ${ }^{10} \mathrm{~A}$ recommendation from a maternity care provider has been shown to be a key factor in increasing vaccination rates. ${ }^{11,12}$

In light of the growing evidence that influenza vaccination during pregnancy has benefits for both the mother and the infant, ${ }^{13-18}$ we evaluated rates of seasonal influenza vaccination among
Competing interests: Jeffrey Scott received payment to attend a medical panel reviewing control strategies for meningococcal disease sponsored by Sanofi Pasteur. Shelly McNeil has conducted clinical trials for manufacturers of influenza vaccines (Sanofi,

GlaxoSmithKline, Novartis) and has received research funding from Sanofi and GlaxoSmithKline. No other competing interests were declared.

This article has been peer reviewed.

Correspondence to:

Linda Dodds,

1.dodds@dal.ca

CMAJ 2014. DOI:10.1503 /cmaj.130499 
pregnant women in the 2 nonpandemic influenza seasons (2010/11 and 2011/12) following the 2009 H1N1 pandemic. We also assessed whether neonatal outcomes differed between women who received the vaccine during pregnancy and those who did not.

\section{Methods}

\section{Study design and population}

We conducted a population-based retrospective cohort study in the province of Nova Scotia. We included all women who delivered a liveborn or stillborn infant at any hospital in the province between Nov. 1, 2010, and Mar. 31, 2012. Women were included if the infant's birth weight was at least $500 \mathrm{~g}$ and the delivery was at 20 weeks' gestation or later.

Approval for the study was received from the IWK Health Centre Research Ethics Board.

\section{Data collection}

We obtained all information from the Nova Scotia Atlee Perinatal Database. Data are collected for the database after hospital discharge from standardized clinical forms and hospital records. Data quality is assessed during periodic reabstraction studies and other validation projects. ${ }^{19,20}$ As of Nov. 1, 2009, the database began collecting information from prenatal records and the hospital admission records on the receipt of influenza vaccine during the current pregnancy. The database also collects data on maternal comorbid factors for serious influenza, including pre-existing diabetes, pulmonary disease, renal disease, heart disease or anemia during pregnancy. ${ }^{5}$ We considered women to be at high risk for serious influenza if they had one or more of these conditions and low risk if they had none.

We also used the Nova Scotia Atlee Perinatal Database to obtain data on potential confounders, including maternal age, body mass index before pregnancy, marital status, education level, neighbourhood income level, smoking status, parity, presence of psychiatric conditions, one or more children under the age of 5, previous pregnancy loss, infant sex and urban versus rural residency.

\section{Outcome measures}

The primary outcomes were preterm birth, low birth weight and small-for-gestational age. The secondary outcomes were low birth weight at term and a composite neonatal mortality and morbidity variable. Data on the following outcomes were obtained from the Nova Scotia Atlee Perinatal Database: fetal death, low birth weight $(<2500 \mathrm{~g})$, small-for-gestational age (defined as the bottom 10th percentile of birth weight [for each sex] for each week of gestational age) and preterm birth (defined as < 37 weeks' gestation at delivery). The composite neonatal mortality and morbidity variable was generated based on a diagnosis of any of the following: neonatal death up to 28 days, low 5-minute Apgar score $(<4)$, sepsis (positive blood culture, septicemia or systemic infection), asphyxia, respiratory distress syndrome (moderate or severe), intraventricular hemorrhage (grade 3 or 4) or acute necrotizing enterocolitis.

\section{Statistical analysis}

We used the $\chi^{2}$ test to compare characteristics between women who received the seasonal influenza vaccine and those who did not. We then used a backward stepwise approach to determine which maternal characteristics were significantly associated with vaccine receipt. The final model included maternal characteristics that, when removed, caused a significant change in the likelihood ratio for the model $(p<0.05)$. We used a complete case analysis, whereby we excluded women for whom data were missing for a covariate included in the final model.

For the analysis of neonatal outcomes, we considered only singleton liveborn infants. For each outcome, we used logistic regression models to estimate odds ratios (ORs) and 95\% confidence intervals (CIs). Initially, unadjusted ORs were calculated for all outcomes. We then used multivariable regression to calculate ORs adjusted for potential confounding variables. The potential confounders were maternal characteristics found to be significantly associated with vaccine receipt in the regression model described earlier. We examined the interaction between vaccine receipt and infant sex, and between vaccine receipt and maternal obesity, to determine whether the ORs for vaccine receipt differed by infant sex or by maternal obesity.

Preterm birth is a time-dependent outcome measure. Because women who deliver preterm have a shorter gestation, they have less opportunity to receive the influenza vaccine during pregnancy than those delivering at term. In a timeinvariant analysis, this creates a potential bias in the direction of showing a protective effect of vaccination on preterm birth. ${ }^{21}$ We attempted to evaluate this bias in 2 ways. First, to determine whether there was a difference in the association between vaccination status and deliveries in November or December versus the other months, we added an interaction term between these 2 variables to the logistic regression model to test its significance. We hypothesized that November and December represent the peak time of 
Table 1 (part 1 of 2): Baseline characteristics of participants according to whether they received seasonal influenza vaccine during pregnancy

\begin{tabular}{|c|c|c|c|c|c|}
\hline \multirow[b]{2}{*}{ Characteristic } & \multirow[b]{2}{*}{$\begin{array}{c}\text { Missing data, } \\
\text { no. (\%) }\end{array}$} & \multicolumn{2}{|c|}{ Vaccination status; no. of women } & \multirow[b]{2}{*}{$\begin{array}{l}\text { Vaccination } \\
\text { rate, } \%\end{array}$} & \multirow[b]{2}{*}{$p$ value* } \\
\hline & & $\begin{array}{l}\text { No vaccine } \\
n=10265\end{array}$ & $\begin{array}{l}\text { Vaccine } \\
n=1958\end{array}$ & & \\
\hline Age, yr & 0 & & & & 0.7 \\
\hline$<20$ & & 598 & 114 & 16.0 & \\
\hline $20-29$ & & 4935 & 920 & 15.7 & \\
\hline $30-34$ & & 3059 & 584 & 16.0 & \\
\hline$\geq 35$ & & 1673 & 340 & 16.9 & \\
\hline Body mass index & $2378(19.5)$ & & & & 0.5 \\
\hline$<18.5$ (underweight) & & 414 & 71 & 14.6 & \\
\hline 18.5-24.9 (normal) & & 3964 & 812 & 17.0 & \\
\hline 25-29.9 (overweight) & & 1900 & 384 & 16.8 & \\
\hline$\geq 30$ (obese) & & 1900 & 400 & 17.4 & \\
\hline Marital status & $645 \quad(5.3)$ & & & & 0.003 \\
\hline Married or common law & & 6947 & 1421 & 17.0 & \\
\hline Single, widowed or divorced & & 2739 & 471 & 14.7 & \\
\hline Education level & $6155(50.4)$ & & & & 0.1 \\
\hline $\begin{array}{l}\text { 1-3 (high school or } \\
\text { technical college) }\end{array}$ & & 1920 & 498 & 20.6 & \\
\hline 4-7 (postsecondary or higher) & & 2955 & 695 & 19.0 & \\
\hline Location of residence & 0 & & & & 0.02 \\
\hline Urban & & 7417 & 1362 & 15.5 & \\
\hline Rural & & 2848 & 596 & 17.3 & \\
\hline Neighbourhood income & $986 \quad(8.1)$ & & & & 0.03 \\
\hline Lowest quintile 1,2 & & 3837 & 686 & 15.2 & \\
\hline Highest quintile ${ }^{3-5}$ & & 5591 & 1123 & 16.7 & \\
\hline Smoking during pregnancy & $92(0.8)$ & & & & $<0.001$ \\
\hline No & & 7951 & 1607 & 16.8 & \\
\hline Yes & & 2228 & 345 & 13.4 & \\
\hline Parity & 0 & & & & 0.01 \\
\hline 0 & & 4706 & 959 & 16.9 & \\
\hline$\geq 1$ & & 5559 & 999 & 15.2 & \\
\hline Season of delivery & 0 & & & & 0.02 \\
\hline November 2010-March 2011 & & 2868 & 575 & 16.7 & \\
\hline April-October 2011 & & 4532 & 796 & 14.9 & \\
\hline November-March 2012 & & 2865 & 587 & 17.0 & \\
\hline Maternal risk statust & 0 & & & & $<0.001$ \\
\hline Low & & 8037 & 1460 & 15.4 & \\
\hline High & & 2228 & 498 & 18.3 & \\
\hline Previous pregnancy loss & $124(1.0)$ & & & & 0.5 \\
\hline No & & 10017 & 1923 & 16.1 & \\
\hline Yes $(\geq 1)$ & & 137 & 22 & 13.8 & \\
\hline$\geq 1$ child at home aged $<5 \mathrm{yr}$ & 0 & & & & 0.7 \\
\hline No & & 6318 & 1213 & 16.1 & \\
\hline \multirow[t]{2}{*}{ Yes } & & 3947 & 745 & 15.9 & \\
\hline & & & & & Continued \\
\hline
\end{tabular}


Table 1 (part 2 of 2): Baseline characteristics of participants according to whether they received seasonal influenza vaccine during pregnancy

\begin{tabular}{|c|c|c|c|c|c|}
\hline \multirow[b]{2}{*}{ Characteristic } & \multirow[b]{2}{*}{$\begin{array}{c}\text { Missing data, } \\
\text { no. (\%) }\end{array}$} & \multicolumn{2}{|c|}{ Vaccination status; no. of women } & \multirow[b]{2}{*}{$\begin{array}{l}\text { Vaccination } \\
\text { rate, } \%\end{array}$} & \multirow[b]{2}{*}{$p$ value } \\
\hline & & $\begin{array}{l}\text { No vaccine } \\
n=10265\end{array}$ & $\begin{array}{c}\text { Vaccine } \\
n=1958\end{array}$ & & \\
\hline Gestational diabetes & 0 & & & & 0.8 \\
\hline No & & 9754 & 1864 & 16.0 & \\
\hline Yes & & 511 & 94 & 15.5 & \\
\hline Gestational hypertension & 0 & & & & 0.1 \\
\hline No & & 10110 & 1938 & 16.1 & \\
\hline Yes & & 155 & 20 & 11.4 & \\
\hline Pre-existing hypertension & 0 & & & & 0.2 \\
\hline No & & 10151 & 1943 & 16.1 & \\
\hline Yes & & 114 & 15 & 11.6 & \\
\hline Any psychiatric condition & 0 & & & & 0.02 \\
\hline No & & 9148 & 1710 & 15.7 & \\
\hline Yes & & 1117 & 248 & 18.2 & \\
\hline No. of fetuses & 0 & & & & 0.7 \\
\hline 1 & & 10093 & 1928 & 16.0 & \\
\hline$\geq 2$ & & 172 & 30 & 14.9 & \\
\hline Sex of infant & 0 & & & & 0.7 \\
\hline Female & & 5024 & 968 & 16.2 & \\
\hline Male & & 5241 & 990 & 15.9 & \\
\hline
\end{tabular}

influenza vaccine receipt and that deliveries during this period are therefore most likely to be affected by this bias. Second, we performed subgroup analyses whereby we repeated the primary analysis but excluded all deliveries taking place during November and December to determine whether similar results would be obtained.

\section{Results}

Of the 12223 women who gave birth to a liveborn or stillborn infant during the study period, $1958(16.0 \%)$ received the seasonal influenza vaccine during their pregnancy. Table 1 shows the demographic and clinical characteristics of the cohort according to whether the vaccine was received or not. Several maternal characteristics were found to be significantly associated with receipt of the seasonal influenza vaccine in the multivariable regression analysis (Table 2). The odds of receiving the vaccine were higher among pregnant women who resided in rural areas and among those with medical comorbidities. Single women, multiparous women and women who smoked during their pregnancy were less likely to have been vaccinated during their pregnancy.

Neonatal outcomes according to maternal vacci- nation status are shown in Table 3 . For this analysis, we included 11293 singleton liveborn infants. In the unadjusted analysis, vaccinated mothers had a significantly lower odds of having a preterm birth (OR $0.75,95 \%$ CI 0.60-0.93) or low-birth-weight infant (OR 0.71, 95\% CI 0.54-0.92) compared with women who did not receive the seasonal influenza vaccine during their pregnancy. The associations between vaccine receipt and the other neonatal outcomes were not statistically significant. The results were unchanged after we adjusted for the potential confounding variables identified in Table 2. The interactions between maternal obesity and receipt of the seasonal vaccine and between infant sex and vaccine receipt were not significant $(p>0.05)$ for any of the outcome measures. Associations between neonatal outcomes and other maternal characteristics are shown in Appendix 1 (available at www.cmaj.ca/lookup/suppl/doi:10 .1503/cmaj.130499/-/DC1).

The interaction term between vaccination status and month of delivery (November and December v. all other months) was not statistically significant for the outcome of preterm birth. In the subgroup analysis in which we excluded deliveries occurring during the peak vaccination periods (November-December 2010 
and 2011), the OR for preterm birth was 0.71 (95\% CI 0.54-0.94), which was similar to the result from the primary analysis.

\section{Interpretation}

The annual rate of seasonal influenza vaccination among pregnant women in Nova Scotia during the 2 nonpandemic influenza seasons immediately following the H1N1 pandemic was a disappointing $16 \%$. This rate is substantially lower than previously reported rates for the $2009 \mathrm{H} 1 \mathrm{~N} 1$ pandemic. Government data suggest that $64 \%$ of pregnant women in Nova Scotia received the H1N1 vaccine during the pandemic. ${ }^{7}$ The rate from our study is similar to rates of seasonal influenza vaccination among pregnant women in the province in the years immediately preceding the pandemic. ${ }^{6}$

In our cohort, women who had received the seasonal influenza vaccine during their pregnancy were less likely to have a preterm birth or lowbirth-weight infant than women who had not received the vaccine. Rates of low-birth-weight infants delivered at term were similar between the vaccinated and nonvaccinated groups, which suggests that the increased likelihood of a low-birthweight infant in the vaccinated group was driven predominantly by the greater likelihood of preterm birth. Our results were essentially unchanged after we adjusted for various maternal characteristics, which suggests that these variables were not actual confounders of the association between vaccine receipt and neonatal outcomes.

It is possible that the mothers of preterm infants had less opportunity to be vaccinated during their pregnancy than mothers of term infants had. However, the results of a sensitivity analysis in which we excluded all deliveries during the peak vaccination period (November and Decem- ber), when the bias would be expected to have an impact, were similar to the results of the primary analysis. In addition, introduction of an interaction term between vaccination status and season of delivery into our regression model for preterm birth had no effect. Given the results of these additional analyses, we do not think that our reported results for preterm birth among vaccinated mothers are influenced by this potential bias.

A study conducted by the US Centers for Disease Control and Prevention (CDC) estimated the rate of seasonal influenza vaccination among pregnant women in the United States during the 2011/12 influenza season was 47\%.22 Other American studies consistently reported similar rates among pregnant women. ${ }^{23}$ In the CDC study, $43 \%$ of women reported receiving both a recommendation and an offer of vaccination by their health care provider. ${ }^{22}$ Vaccination rates among these women were significantly increased (74\%). Among women who received neither a recommendation nor an offer of vaccination, the rate was only $11 \% .^{22}$ Several studies have similarly shown strong asso-

Table 2: Factors associated with receipt of seasonal influenza vaccine during pregnancy

\begin{tabular}{|lc|}
\hline Factor & Adjusted OR* $(95 \% \mathrm{Cl})$ \\
\hline High-risk statust (v. low risk) & $1.22(1.08-1.38)$ \\
\hline Smoking during pregnancy (v. no smoking) & $0.81(0.71-0.94)$ \\
\hline $\begin{array}{l}\text { Single, widowed or divorced (v. married or } \\
\text { common law) }\end{array}$ & $0.83(0.07-0.94)$ \\
\hline Multiparous (v. primiparous) & $0.87(0.78-0.96)$ \\
\hline Rural residence (v. urban) & $1.18(1.06-1.32)$ \\
\hline $\begin{array}{l}\text { Note: Cl = confidence interval, OR = odds ratio. } \\
\text { *Adjusted for other terms in the model. } \\
\text { tPre-existing diabetes, pulmonary disease (including asthma), renal disease, heart disease or } \\
\text { anemia. }\end{array}$ \\
\hline
\end{tabular}

Table 3: Association between neonatal outcomes among singleton liveborn infants $(n=11293)$ and mother's receipt of seasonal influenza vaccine during pregnancy

\begin{tabular}{|c|c|c|c|c|}
\hline \multirow[b]{2}{*}{ Outcome } & \multicolumn{2}{|c|}{$\begin{array}{c}\text { Maternal vaccination status; } \\
\text { no. }(\%) \text { of infants }\end{array}$} & \multicolumn{2}{|c|}{ Effect of vaccine (v. no vaccine) } \\
\hline & $\begin{array}{l}\text { No vaccine } \\
n=9437^{\star}\end{array}$ & $\begin{array}{l}\text { Vaccine } \\
n=1856^{*}\end{array}$ & $\begin{array}{l}\text { Unadjusted OR } \\
\quad(95 \% \mathrm{Cl})\end{array}$ & $\begin{array}{c}\text { Adjusted } \\
\text { ORt }(95 \% \mathrm{Cl})\end{array}$ \\
\hline Preterm birth (< 37 wk) & $617(6.5)$ & $92(5.0)$ & $0.75(0.60-0.93)$ & $0.75(0.60-0.94)$ \\
\hline Low birth weight $(<2500 \mathrm{~g})$ & $461(4.9)$ & $65(3.5)$ & $0.71(0.54-0.92)$ & $0.73(0.56-0.95)$ \\
\hline Low birth weight at term & $160(1.7)$ & $26(1.4)$ & $0.82(0.54-1.25)$ & $0.85(0.56-1.29)$ \\
\hline Small-for-gestational age ( $<10$ th percentile) & $749(8.0)$ & $138(7.5)$ & $0.93(0.77-1.12)$ & $0.96(0.79-1.16)$ \\
\hline Composite neonatal morbidity variable $\neq$ & $441(4.7)$ & $95(5.1)$ & $1.10(0.88-1.38)$ & $1.06(0.85-1.34)$ \\
\hline \multicolumn{5}{|c|}{$\begin{array}{l}\text { Note: } \mathrm{Cl}=\text { confidence interval, } \mathrm{OR}=\text { odds ratio. } \\
\text { *Numbers exclude infants whose mothers had missing values for adjustment covariates in the model. } \\
\text { tAdjusted for maternal age, high-risk status, smoking during pregnancy, marital status, parity and location of residence. } \\
\text { flncludes neonatal death, asphyxia, sepsis, low Apgar score at } 5 \text { min, moderate or severe respiratory distress syndrome, intraventricular hemorrhage and acute } \\
\text { necrotizing enterecolitis. }\end{array}$} \\
\hline
\end{tabular}


ciations between recommendations by care providers and increased likelihood of vaccination. ${ }^{89,23,24}$ A lack of knowledge of the risks of influenza during pregnancy and concerns about the fetal safety of the vaccine are commonly cited barriers to influenza vaccination during pregnancy. ${ }^{8,923}$ However, it has been shown that, at the recommendation of their care provider, women will often agree to vaccination despite their concerns. ${ }^{24}$

A growing number of studies have addressed barriers to influenza vaccination during pregnancy and potential interventions to help improve vaccine uptake. Despite strong evidence showing the importance of recommendations by care providers, less than half of pregnant women in 2 recent US studies reported receiving this advice. ${ }^{22,23}$ Studies evaluating the efficacy of provider-focused reminders to offer vaccination to pregnant patients have been successful at significantly increasing vaccination rates, ${ }^{25,26}$ which suggests that this is a promising strategy. In contrast, a randomized study involving patient reminders sent via text messaging failed to improve vaccination rates. ${ }^{27}$

Observational studies investigating the association between maternal influenza vaccination and preterm birth have yielded conflicting results thus far. In an American cohort study, comparison of vaccinated and nonvaccinated mothers during the $2009 \mathrm{H} 1 \mathrm{~N} 1$ pandemic showed an association between vaccine receipt and reduced likelihood of preterm birth. ${ }^{28}$ In a study conducted during the 2009 pandemic in Ontario, Canada, Fell and colleagues showed an association between $\mathrm{H} 1 \mathrm{~N} 1$ vaccination and reduced likelihood of very-preterm birth $(<32$ wk gestation).$^{29}$ For nonpandemic influenza seasons, the majority of studies have not shown an association between seasonal influenza vaccination during pregnancy and subsequent risk of premature birth. ${ }^{6,18,30}$ However, a cohort study by Omer and colleagues ${ }^{17}$ that analyzed 4326 live births occurring between 2004 and 2006 found that infants born during the influenza season whose mothers received the seasonal influenza vaccine were significantly less likely to be premature than infants born to nonvaccinated women during the same period. It is unclear whether the inconsistent findings reported thus far may be due to a true lack of association, or whether they may be the result of other methodologic challenges (e.g., small samples) or possibly differences in influenza epidemiology or demographic characteristics between study populations.

Although the cause of preterm birth is complex, maternal infection has been identified as a risk factor. ${ }^{31}$ It is hypothesized that the link between infection and prematurity is mediated in part by inflammatory pathways. Increases in the pro-inflammatory cytokines interleukin- 1 and tumour necrosis factor- $\alpha$ have been shown to stimulate the production of prostaglandins in the amnion and decidua. ${ }^{32,33}$ Prostaglandins play a key role in the initiation of labour, mainly through the stimulation of uterine contractility. ${ }^{34}$ To further support this inflammation theory of preterm birth, animal models have shown that the administration of either interleukin-1 or tumour necrosis factor- $\alpha$ can induce preterm labour. ${ }^{32,33}$ In recent studies, influenza virus infection has been specifically shown to induce gene expression of various pro-inflammatory cytokines, including interleukin-1 and tumour necrosis factor- $\alpha .{ }^{32}$ By averting maternal infection, the vaccine may exert a protective effect on the fetus by avoiding the associated inflammatory response that may trigger premature labour.

Few studies have evaluated neonatal outcomes associated with maternal seasonal influenza vaccination, and all were conducted before the H1N1 pandemic. ${ }^{6,17,18}$ Two studies showed a reduced likelihood of preterm birth and small-for-gestational age, but only in periods of widespread influenza activity; ${ }^{17,18}$ the third study observed a reduced risk of low birth weight and small-for-gestational age associated with influenza vaccination. ${ }^{6}$ As well, several recent studies have shown reductions in influenza-related illness among infants whose mothers received the seasonal influenza vaccine during pregnancy..$^{13-16}$ Our population-based study of the impact of maternal seasonal influenza vaccination on neonatal outcomes after the H1N1 pandemic adds to the existing evidence that suggests a benefit of maternal influenza vaccination for the infant.

\section{Limitations}

Although the Nova Scotia Atlee Perinatal Database has been validated in several studies, its collection of data on maternal influenza vaccination is relatively new and those data have not yet been validated. However, the government of Nova Scotia reported a vaccination rate among pregnant women of $18.9 \%$ for the 2010/11 influenza season, ${ }^{35}$ which agrees quite closely with our estimated rate during this period. A number of studies have assessed the accuracy of self-reported influenza vaccination history and found it to be acceptable, although most have been specific to populations of older people. ${ }^{36,37}$

The relatively low number of deliveries occurring in Nova Scotia each year prevented analyses of rare outcomes such as fetal death and neonatal death. Because we report multiple outcome measures, some of our associations may have been the result of a type I error. The availability of information on maternal demographic 
characteristics, health behaviours and medical history in the Atlee Perinatal Database supported the assessment of a number of potential confounding variables. Other potential confounders such as ethnic background and maternal education were not available, and it is possible that these, or other variables, were confounding the association between receipt of influenza vaccine and pregnancy outcomes.

Another limitation of our study is the lack of information regarding date of maternal vaccination, which prevented the analysis of trimesterspecific effects of vaccination. Date of vaccination would also have allowed exclusion of term infants whose mothers received the vaccination after 37 weeks' gestation from our analysis of preterm birth.

\section{Conclusion}

Our population-based study showed a reduced likelihood of preterm birth and low birth weight among infants whose mothers received the seasonal influenza vaccine during pregnancy. Our findings add to the existing body of evidence that showing that seasonal influenza vaccination during pregnancy not only offers maternal benefits, but may also provide both prenatal benefits to the fetus and postnatal protection to the infant through transplacental antibodies. Given the mounting evidence, both Canadian and World Health Organization guidelines now recommend routine seasonal influenza vaccination of all pregnant women in any trimester. ${ }^{5,38}$

However, despite these recommendations, the rates of seasonal influenza vaccination in our study were disappointingly low. Our attention must now turn toward efforts to improve these vaccination rates. Thus far, the evidence suggests that rates are highest when pregnant women receive a recommendation for influenza vaccination from their antenatal care provider, who can then offer the vaccine in the same care setting. Future research should include comparisons of vaccination rates between different care models. Once the care models with optimal vaccine uptake are identified, research will be needed into how best to incorporate them into practice.

\section{References}

1. Neuzil KM, Reed GW, Mitchel EF, et al. Impact of influenza on acute cardiopulmonary hospitalizations in pregnant women. Am J Epidemiol 1998;148:1094-102.

2. Schanzer DL, Langley JM, Tam TW. Influenza-attributed hospitalization rates among pregnant women in Canada 1994-2000. J Obstet Gynaecol Can 2007;29:622-9.

3. Dodds L, McNeil SA, Fell DB, et al. Impact of influenza exposure on rates of hospital admissions and physician visits because of respiratory illness among pregnant women. CMAJ 2007;176: 463-8.

4. McNeil SA, Dodds LA, Fell DB, et al. Effect of respiratory hospitalization during pregnancy on infant outcomes. Am J Obstet Gynecol 2011;204:S54-7.
5. National Advisory Committee on Immunization. Statement on seasonal trivalent inactivated influenza vaccine (TIV) for 2009 2010. Can Commun Dis Rep 2009;35:1-41.

6. Dodds L, MacDonald N, Scott J, et al. The association between influenza vaccine in pregnancy and adverse neonatal outcomes. J Obstet Gynaecol Can 2012;34:714-20.

7. Nova Scotia's response to H1N1: summary report. Halifax (NS): Government of Nova Scotia; 2010. Available: www.gov.ns.ca /dhw/publications/H1N1-Summary-Report.pdf (accessed 2013 Feb. 20).

8. Fisher BM, Scott J, Hart J, et al. Behaviors and perceptions regarding seasonal and $\mathrm{H} 1 \mathrm{~N} 1$ influenza vaccination during pregnancy. Am J Obstet Gynecol 2011;204:S107-11.

9. Yudin MH, Salaripour M, Sgro MD. Pregnant women's knowledge of influenza and the use and safety of the influenza vaccine during pregnancy. J Obstet Gynaecol Can 2009;31:120-5.

10. Bednarczyk RA, Adjaye-Gbewonyo D, Omer SB. Safety of influenza immunization during pregnancy for the fetus and neonate. Am J Obstet Gynecol 2012;207(Suppl):S38-46.

11. Tong A, Biringer A, Ofner-Agostini M, et al. A cross-sectional study of maternity care providers' and women's knowledge, attitudes, and behaviours towards influenza vaccination during pregnancy. J Obstet Gynaecol Can 2008;30:404-10.

12. Steelfisher GK, Blendon RJ, Bekheit MM, et al. Novel pandemic A (H1N1) influenza vaccination among pregnant women: motivators and barriers. Am J Obstet Gynecol 2011;204:S116-23.

13. Zaman K, Roy E, Arifeen SE, et al. Effectiveness of maternal influenza immunization in mothers and infants [published erratum in N Engl J Med 2009;360:648]. N Engl J Med 2008;359:1555-64.

14. Benowitz I, Esposito DB, Gracey KD, et al. Influenza vaccine given to pregnant women reduces hospitalization due to influenza in their infants. Clin Infect Dis 2010;51:1355-61.

15. Eick AA, Uyeki TM, Klimov A, et al. Maternal influenza vaccination and effect on influenza virus infection in young infants. Arch Pediatr Adolesc Med 2011;165:104-11.

16. Poehling KA, Sziliagyi PG, Staat MA, et al. Impact of maternal immunization on influenza hospitalizations in infants. Am J Obstet Gynecol 2011;204:S141-8.

17. Omer SB, Goodman D, Steinhoff MC, et al. Maternal influenza immunization and reduced likelihood of prematurity and small for gestational age births: a retrospective cohort study. PLoS Med 2011;8:e1000441.

18. Steinhoff MC, Omer SB, Roy E, et al. Influenza immunization in pregnancy - antibody responses in mothers and infants. N Engl J Med 2010;362:1644-6.

19. Fair M, Cyr M, Allen AC, et al. An assessment of the validity of a computer system for probabilistic record linkage of birth and infant death records in Canada. Chronic Dis Can 2000;21:8-13.

20. Joseph KS, Fahey J. Validation of perinatal data in the Canadian Institute for Health Information's Discharge Abstract Database. Chronic Dis Can 2009;29:96-100.

21. Ahrens K, Lash TL, Louik C, et al. Correcting for exposure misclassification using survival analysis with a time-varying exposure. Ann Epidemiol 2012;22:799-806.

22. US Centers for Disease Control and Prevention. Influenza vaccination coverage among pregnant women: 2011-12 influenza season, United States. MMWR Morb Mortal Wkly Rep 2012;61:758-63.

23. Henninger M, Naleway A, Crane B, et al. Predictors of seasonal influenza vaccination during pregnancy. Obstet Gynecol 2013; 121:741-9.

24. Wiley KE, Massey PD, Cooper SC, et al. Uptake of influenza vaccine by pregnant women: a cross-sectional survey. Med J Aust 2013;198:373-5

25. Sherman MJ, Raker CA, Phipps MG. Improving influenza vaccination rates in pregnant women. J Reprod Med 2012;57:371-6.

26. Klatt TE, Hopp E. Effect of a best-practice alert on the rate of influenza vaccination of pregnant women. Obstet Gynecol 2012; 119:301-5.

27. Moniz MH, Hasley S, Meyn LA, et al. Improving influenza vaccination rates in pregnancy through test messaging: a randomized controlled trial. Obstet Gynecol 2013;121:734-40.

28. Richards JL, Hansen C, Bredfeldt C, et al. Neonatal outcomes after antenatal influenza immunization during the $2009 \mathrm{H} 1 \mathrm{~N} 1$ influenza pandemic: impact on preterm birth, birth weight, and small for gestational age birth. Clin Infect Dis 2013;56:1216-22.

29. Fell DB, Sprague AE, Liu N, et al. H1N1 influenza vaccination during pregnancy and fetal and neonatal outcomes. Am J Public Health 2012;102:e33-40.

30. Black SB, Shinefield HR, France EK, et al. Effectiveness of influenza vaccine during pregnancy in preventing hospitalizations and outpatient visits for respiratory illness in pregnant women and their infants. Am J Perinatol 2004;21:333-9.

31. Goldenberg RL, Culhane JF, Iams JD, et al. Epidemiology and causes of preterm birth. Lancet 2008;371:75-84. 
32. Uchide N, Ohyama K, Bessho T, et al. Induction of proinflammatory cytokine gene expression and apoptosis in human chorion cells of fetal membranes by influenza virus infection: possible implications for maintenance and interruption of pregnancy during infection. Med Sci Monit 2005;11:RA7-16.

33. Romero R, Durum S, Dinarello CA, et al. Interleukin-1 stimulates prostaglandin biosynthesis by human amnion. Prostaglandins 1989;37:13-22.

34. Challis JR, Sloboda DM, Alfaidy N, et al. Prostaglandins and mechanisms of preterm birth. Reproduction 2002;124:1-17.

35. Influenza: surveillance report 2011-2012 influenza season. Halifax (NS): Nova Scotia Department of Health and Wellness; 2012. Available: http://novascotia.ca/dhw/populationhealth/documents /Influenza_Surveillance_Report_2011-2012.pdf (accessed 2013 Dec 9).

36. Rolnick SJ, Parker ED, Nordin JD, et al. Self-report compared to electronic medical record across eight adult vaccines: Do results vary by demographic factors? Vaccine 2013;31:3928-35.

37. Zimmerman RK, Raymund M, Janosky JE, et al. Sensitivity and specificity of patient self-report of influenza and pneumococcal polysaccharide vaccinations among elderly outpatients in diverse patient care strata. Vaccine 2003;21:1486-91.

38. Vaccines against influenza: WHO position paper - November 2012. Wkly Epidemiol Rec 2012;87:461-76.
Affiliations: Departments of Obstetrics and Gynaecology (Legge, Dodds), Pediatrics (Legge, Dodds, MacDonald, Scott) and Medicine (McNeil), Dalhousie University, Halifax, NS; Canadian Center for Vaccinology (Dodds, MacDonald, Scott, McNeil), IWK Health Centre and Capital Health, Halifax, NS

Contributors: Linda Dodds, Noni MacDonald and Shelly McNeil conceived the study design, and Alexandra Legge and Linda Dodds developed the study protocol. Alexandra Legge and Linda Dodds analyzed the data, and all of the authors contributed to the interpretation of the data. Alexandra Legge wrote the first draft of this manuscript, and all of the authors assisted in revising it. All of the authors approved the final version of the manuscript submitted for publication.

Funding: No direct funding was received for this study. Alexandra Legge was supported by a Dr. Carl Tupper Summer Studentship from the Dalhousie Medical Research Foundation.

Acknowledgement: The authors thank the Reproductive Care Program of Nova Scotia for access to the data. 